\title{
Absolute determination of the wavelength and spectrum of an extreme-ultraviolet beam by a Young's double-slit measurement
}

\author{
R. A. Bartels, A. Paul, M. M. Murnane, H. C. Kapteyn, and S. Backus \\ JILA, University of Colorado and National Institute of Standards and Technology, Boulder, Colorado 80309-0440 \\ Y. Liu and D. T. Attwood \\ Center for X-Ray Optics, Lawrence Berkeley National Laboratory, and Applied Science and Technology Graduate Group, \\ University of California, Berkeley, Berkeley, California 94720
}

Received November 18, 2001

\begin{abstract}
The interference pattern produced by irradiation of a pair of pinholes with a beam contains information on both the spatial and the temporal coherence properties of the beam, as well as its power spectrum. We demonstrate experimentally for what is believed to be the first time that the spectrum of an extreme-ultraviolet (EUV) beam can be obtained from a measurement of the interference pattern produced by a pinhole pair. This approach offers a convenient method of making absolute wavelength and relative spectral intensity calibrations in the EUV. (C) 2002 Optical Society of America

OCIS codes: $\quad 030.1670,260.3160,260.7200,260.1960,070.4790,300.6170$.
\end{abstract}

Although Young performed his original double-slit experiment 200 years ago, ${ }^{1}$ it continues to yield new insights into the properties of light sources. The interference pattern resulting from a double slit (or pinhole pair) is commonly used to measure the spatial coherence properties of a light source in the visible ${ }^{2}$ as well as in the extreme-ultraviolet ${ }^{3,4}$ (EUV) regions of the spectrum. This interference pattern results from the path difference of the incident light field from each slit to the observation point. In a standard coherence measurement the incident field is assumed to be quasi monochromatic, so temporal coherence effects are isolated from a measurement of spatial coherence. $^{5}$ However, under conditions in which a broad-bandwidth source illuminates a pinhole pair, the interference pattern will contain both temporal and spatial coherence information on the source as well as the power spectrum. ${ }^{6}$ In this Letter we demonstrate that the power spectrum of a coherent EUV beam, consisting of four harmonic orders generated by high-harmonic emission ${ }^{7,8}$ (HHG), can be measured by analysis of the far-field intensity distribution produced by a pinhole pair. Such a spectral measurement can be calibrated by a straightforward measurement of the geometry of the experiment, providing absolute wavelength and relative intensity information. This approach proves particularly useful in the EUV, since wavelength calibration to high accuracy can be achieved with straightforward measurements, and the only element that has a spectral response that must be separately calibrated is the CCD. This experiment is, to our knowledge, the first spectral measurement of a nonmonochromatic source by analysis of the pinhole-pair interference pattern at any wavelength.

Our analysis is based on coherence theory., ${ }^{2,6,6}$ In the far field the path-length difference between two sampled portions of a field $\left[P_{1}\right.$ and $P_{2}$ in Fig. 1(a)] gives rise to an interference pattern that is determined by the spatial and temporal coherence of the source. This path difference also introduces a time delay,
$\tau=\Delta r / c=d x /(z c)$, that generates an autocorrelation, $\int E(t) E^{*}(t-\tau) \mathrm{d} t$, of the incident field. The Wiener-Khinchin theorem shows that the power spectrum is the Fourier transform of the field autocorrelation. ${ }^{5}$ Therefore, measuring the pinhole-pair interference pattern is equivalent to measuring the power spectrum of the field that is incident on the pinhole pair, provided that the field is spatially coherent and the spatial extent of the field autocorrelation is less than the width of the Airy pattern from a single pinhole in the observation plane. This connection has
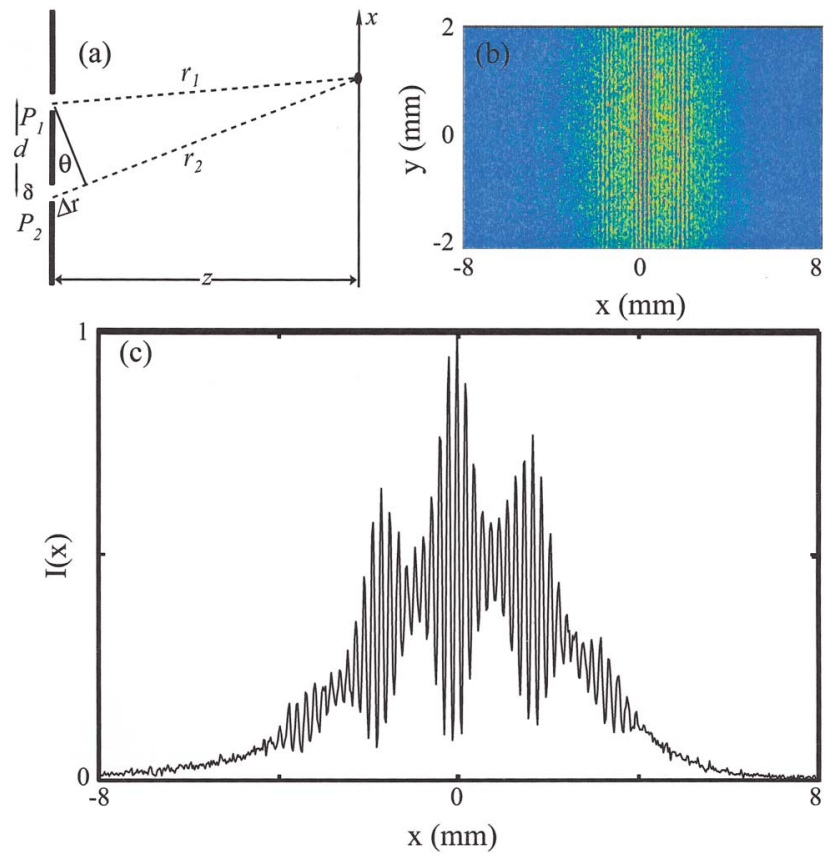

Fig. 1. (a) Schematic of the experimental setup, where we have recorded (b) the EUV interferogram (note that the intensity is weakest for blue and strongest for red colorings), and (c) average of (b) along the $y$ axis. The experimental conditions used here are $z=2.85 \mathrm{~m}, \delta=20 \mu \mathrm{m}$ and $d=574.6 \mu \mathrm{m}$. 
been known for some time ${ }^{6}$ but to date has not been exploited for a determination of the power spectrum of a light field.

The exact relationship between the interference pattern and power spectrum can be derived as follows. The intensity distribution in the observation plane after illumination of two pinholes equally can be written as

$$
I(x)=2 I^{(0)}(x)\left[1+\gamma_{12}(x) \cos \left(2 \pi \frac{d}{\lambda_{0} z} x\right)\right],
$$

where $I^{(0)}(x)$ is the Airy distribution due to diffraction through a pinhole of width $\delta, d$ is the pinhole separation, $z$ is the distance from the pinhole pair to the observation plane, $\lambda_{0}$ is the central wavelength of the light field, and $\gamma_{12}$ is the complex degree of mutual coherence. Here the time delay has been transformed to the spatial coordinate $x=z c \tau / d$. At any particular wavelength, the fringe visibility, ${ }^{5} \mu_{12}=\left[I(x)_{\max }-\right.$ $\left.I(x)_{\min }\right] /\left[I(x)_{\max }+I(x)_{\min }\right]$, is simply the value of $\gamma_{12}$ at $x=\tau=0$, i.e., $\mu_{12}=\gamma_{12}(0)$. More generally, ${ }^{9}$

$$
\gamma_{12}(\tau)=\mathcal{T}^{-1}\left\{\hat{S}(\nu) \mu_{12}(\nu)\right\},
$$

where $\hat{S}(\nu)$ is the power spectrum normalized such that $\int_{0}^{\infty} \hat{S}(\nu) \mathrm{d} \nu=1$. From Eq. (1), the interference pattern from a pinhole pair, shown in Fig. 1(b), will have a broad spatial extent determined by the Airy distribution from a single pinhole. The modulations within the Airy disk are due to interference of the two pinholes. The slow modulations are due to the interference of the broad bandwidth associated with several harmonics, while the fast oscillations are determined by the central wavelength. The depth of modulation is determined by the spatial coherence of the beam, and therefore this technique can also be applied to light fields with imperfect spatial coherence.

Equation (1) is best analyzed in the spatial frequency domain. A Fourier transform of Eq. (1) gives

$$
\begin{aligned}
\mathcal{T}\{I(x)\} \equiv & F\left(f_{x}\right)=2 T\left(f_{x}\right) \otimes\left\{\delta\left(f_{x}\right)+\frac{1}{2} \hat{S}\left(f_{x}\right) \mu_{12}\left(f_{x}\right)\right. \\
& \left.\otimes\left[\delta\left(f_{x}-f_{0}\right)+\delta\left(f_{x}-f_{0}\right)\right]\right\},
\end{aligned}
$$

where $\otimes$ is the convolution operator, $T\left(f_{x}\right)=\mathcal{T}\left\{I^{(0)}(x)\right\}$ is a dc spike, $\delta\left(f_{x}\right)$ is the Dirac delta function, $f_{0}=d /\left(z \lambda_{0}\right)$ is the carrier spatial frequency due to the pinhole-pair interference pattern, and $\hat{S}\left(f_{x}\right) \mu_{12}\left(f_{x}\right)=\mathcal{T}\left\{\gamma_{12}(x)\right\}$. Thus, a Fourier transform of the interferogram produced from a Young's pinholepair measurement should yield three terms: a dc term corresponding to a spike at zero (or dc) frequency and two terms containing information on the power spectrum convolved with the dc spike and weighted by the spatial coherence function at that frequency. As a result, the resolution of a spectral measurement is limited by the width of the dc spike. This resolution limit approximately corresponds to the number of interference fringes that can be resolved under the Airy distribution from a single pinhole and is thus proportional to the diameter of the pinhole and inversely proportional to the pinhole separation. Equation (3) yields information on only the product of the power spectrum and the spatial coherence function at any frequency. However, in the case of HHG generated in a hollow-core fiber, ${ }^{7}$ the four to five harmonics that emerge are phase matched. As a result, we expect the spatial coherence $\mu_{12}(\nu)$ across the entire four-harmonic spectrum to be very high and nearly constant. Recent spatial coherence measurements made under the same conditions, and with the same harmonics irradiating an aperture simultaneously, confirm this assumption. Fringe visibilities of unity were observed over most of the beam, and for this pinhole separation the average coherence as a function of wavelength, $\int_{0}^{\infty} \hat{S}(\nu) \mu_{12}(\nu) \mathrm{d} \nu$, is $\sim 0.9$, indicating that the average spectral deviation of the spatial coherence is $<10^{-2} / \mathrm{nm}$.

For our experiments we used 27-fs laser pulses generated by an amplified Ti:sapphire laser system at a $5-\mathrm{kHz}$ repetition rate, a wavelength of $760 \mathrm{~nm}$, and with energy of $\sim 700 \mu \mathrm{J}^{10}$ The pulse is focused into a 10 -cm-long, $150-\mu \mathrm{m}$-diameter hollow-core fiber filled with 30 Torr of Ar gas such that the HHG process is phase matched and peaked at the 21st harmonic under our conditions. ${ }^{7}$ A $0.35-\mu \mathrm{m}$-thick $\mathrm{Al}$ filter is used to remove the fundamental IR beam, after which a $20-\mu \mathrm{m}$-diameter pinhole pair with a $574.6-\mu \mathrm{m}$ center-to-center separation (as verified by a scanning electron microscope) is placed $95 \mathrm{~cm}$ from the fiber exit. The EUV beam was measured to be $\sim 1 \mathrm{~mm} 1 / e^{2}$ diameter at this location. The far-field diffraction pattern observed $2.85 \mathrm{~m}$ from the pinhole pair with an EUV-sensitive CCD camera (Andor) with an exposure time of $60 \mathrm{~s}$ is shown in Fig. 1(b). An additional $0.35-\mu \mathrm{m}$-thick Al filter placed immediately before the CCD camera eliminates unwanted IR scattered light. The HHG spectra were also measured by an imaging EUV spectrometer (Hettrick Scientific HiREFS SXR-1.75), which measures $\eta(\nu) \hat{S}(\nu)$, where $\eta(\nu)$ is the relative efficiency of the spectrometer.

Figure 2 shows the spatial frequency distribution along the $x$ dimension, obtained from a Fourier

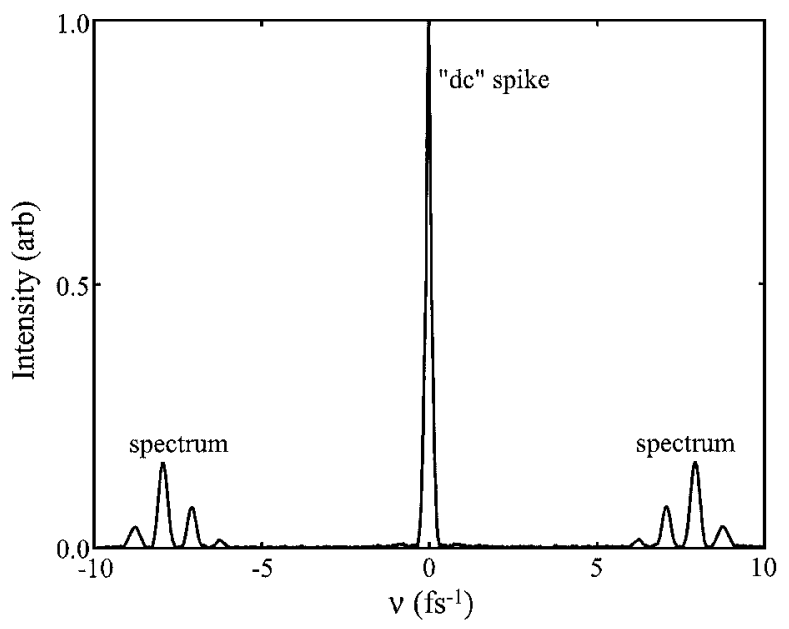

Fig. 2. Fourier transforms of Fig. 1(b), showing the spatial frequency distribution scaled in optical frequency. The central frequency observed in the sidebands corresponds to the $21 \mathrm{st}$ harmonic of the laser at $800 \mathrm{~nm}$. 


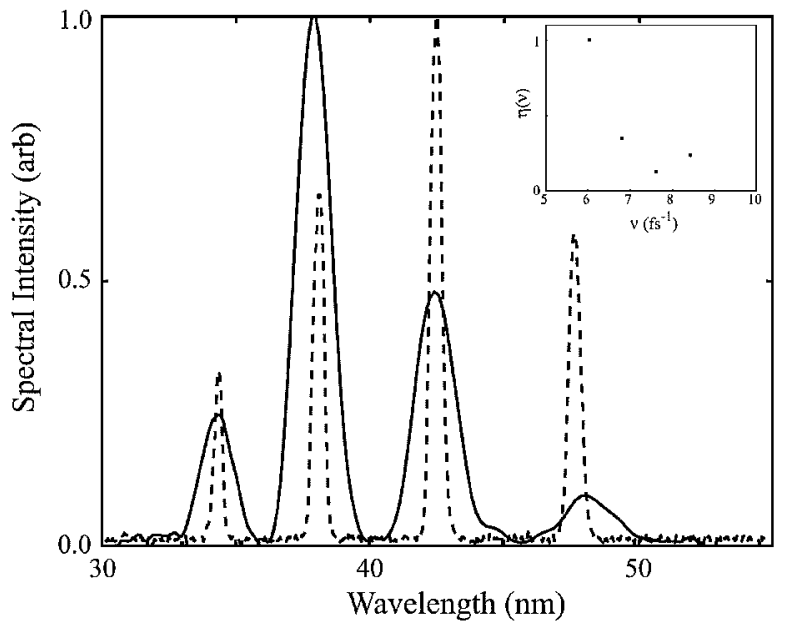

Fig. 3. EUV spectrum obtained from a spectrometer $[\hat{S}(\nu) \eta(\nu)]$ (dashed curve) and from the spatial coherence measurement $[\hat{S}(\nu)]$ (solid curve). The inset shows the spectrometer relative efficiency, $\eta(\nu)$, inferred from these data. When the spectrometer data are convolved with the pinhole resolution, the measured and calculated linewidths agree to within $10 \%$.

transform of the interferogram produced by the pinhole pair [shown in Fig. 1(b)]. The high quality of the data of Fig. 2 is due in large part to the fact that the two-dimensional Fourier transform of the interferogram (with substantial random detector noise) implicitly averages over all 256 lines of data in the $y$ dimension. In essence, we take 256 simultaneous single-shot field autocorrelation traces; the interferogram averaged over all $y$ is shown in Fig. 1(c). The optical frequency axis is obtained by multiplication of the spatial frequency axis by $z c / d$. Therefore, the pinhole separation and the distance to the detector determine the calibration of the wavelength axis in Fig. 3. The three terms expected from Eq. (3) are clearly shown in Fig. 2 . The dc spike appears at the zero spatial frequency and has a fractional width of $\Delta \nu / \nu_{0}=0.022$, which agrees well with the predicted resolving power of $\Delta \nu / \nu_{0}=0.8 \delta / d$, or 0.027 for our experimental geometry.

The percent error in the optical frequency calibration can be written as $\Delta \nu / \nu=\Delta z / z+\Delta d / d$ and demonstrates that the accuracy of the frequency scaling is directly related to the accuracy of the geometry measurement. In this experiment the distance to the detector is measured within $\sim \pm 0.02 \%$ and pinhole separation is measured within $\sim \pm 0.008 \%$, with an overall accuracy of $\sim \pm 0.03 \%( \pm 2 \mathrm{THz})$. Note that it is easy to obtain an accuracy much higher than the resolving power of the instrument. An alternative technique for calibration of the optical frequency is to use a well-characterized, narrow-band optical source (e.g., a $\mathrm{He}-\mathrm{Ne}$ laser) at one wavelength and record the interference fringes, which can be used to calibrate the scaling constant, $z c / d$. Once determined, that constant can be used to accurately calibrate the spectrum of an optical field in any spectral region.

The HHG spectrum appears as two sidebands well separated from the dc term of the spatial frequency distribution. The harmonic peaks are broadened because of the intrinsic resolution of this measurement; i.e., we measure a convolution $T(\nu) \otimes \hat{S}(\nu) \mu_{12}(\nu)$ or $T(\nu) \otimes \hat{S}(\nu)$, assuming constant $\mu_{12}(\nu)$, as is the case for this experiment. The broadening is evident from the comparison of the spectrum measured by the x-ray spectrometer (dashed curve of Fig. 3) compared with the pinhole spectrum (solid curve of Fig. 3). However, the width of the spectrum obtained from the $\mathrm{x}$-ray spectrometer after being convolved with the dc spike is identical to that obtained from the pinhole pair. The difference in the intensity of the two spectra is due to the varying efficiency of spectrometer response, assuming constant spatial coherence across the harmonic spectrum, as explained above. The inset of Fig. 3 plots the ratio of the two spectra, $[\hat{S}(\nu) \eta(\nu) / \hat{S}(\nu)=\eta(\nu)]$. The spectral response, including an $\sim 50-\mathrm{nm} \mathrm{SiO}_{2}$ passivation layer on the CCD chip, must be known for a complete determination of the spectral intensity distribution.

In summary, we have experimentally demonstrated a robust and accurate technique that allows the absolute wavelength and spectrum of a light field to be determined from the far-field interferogram produced by a pinhole pair. Furthermore, this technique provides a convenient method of absolute calibration in the EUV region of the spectrum where few tunable sources currently exist. The spectrum obtained is verified with the spectrum measured by a conventional grating spectrometer. The resolution of the pinhole-pair spectrum can be improved by a simple change in geometry, by either shrinking the pinhole diameter or increasing the pinhole separation, at the cost of reduced photon flux. The quality of the data is extremely high because of the implicit averaging of the two-dimensional Fourier transform. Finally, this experiment provides verification of an important link between the spatial coherence properties of an optical field and its power spectrum.

The authors gratefully acknowledge support for this work from the U.S. Department of Energy. R. Bartels's e-mail address is bartels@jila.colorado.edu.

\section{References}

1. T. Young, Philos. Trans. R. Soc. London 12, 387 (1802).

2. B. Thompson and E. Wolf, J. Opt. Soc. Am. 46, 895 (1957).

3. C. Chang, P. Naulleau, E. Anderson, and D. Attwood, Opt. Commun. 182, 25 (2000).

4. Y. Lui, M. Seminario, F. Tomasel, C. Chang, J. Rocca, and D. Attwood, Phys. Rev. A 63, 033802 (2001).

5. J. Goodmann, Statistical Optics (Wiley, New York, 1985).

6. E. Wolf and A. Devaney, Opt. Lett. 6, 168 (1981).

7. A. Rundquist, C. Durfee, Z. Chang, C. Herne, S. Backus, M. M. Murnane, and H. C. Kapteyn, Science 280, 1412 (1998).

8. C. Durfee, A. Rundquist, S. Backus, C. Herne, M. M. Murnane, and H. C. Kapteyn, Phys. Rev. Lett. 83, 2187 (1999).

9. A. Friberg and E. Wolf, Opt. Lett. 20, 623 (1995).

10. S. Backus, R. Bartels, S. Thompson, R. Dollinger, H. C. Kapteyn, and M. M. Murnane, Opt. Lett. 26, 465 (2001). 